\title{
Tracking Technique for Athletes in Skilled Video
}

\author{
Song Ailing, Chen Kai \\ Southwest Jiaotong University Sports Ministry \\ Chengdu, China
}

\begin{abstract}
Accurate analysis of athletes' video is an effective technical way to improve the training effect, and the realization of tracing of athletes in the skilled video is essential for its analysis. Based on OpenCV (open source computer vision library supported by Intel) technology platform, we realize the athletes tracing in the skilled vide using the combination algorithm of Kalman prediction and CamShift tracing. The case application shows that our technical solution is reasonable and feasible, which can provide the valuable references to the related research.
\end{abstract}

\section{Keywords; Physical education;video;tracing;algorithm}

\section{INTRODUCTION}

Nowadays the rapid development of science and technology promotes the rapid development of competitive sports. Modern high-tech achievements, latest knowledge and technologies are fully used in PE area and play an important part in the promotion of competitive sports ${ }^{[1-3]}$. If they want to get ideal results, athletes must practice scientifically for a long time. So, how to use the correct method and way to evaluate their own characteristics and weaknesses in practice to formulate technical movements training method and set up a complete set of coherent training system, is very important.

Combined with domestic and foreign literature, in order to get the best training result, nowadays many of the world' s outstanding coaches fully use various modern science and technology in the training process, to enhance the training target and purposiveness and to make the training develop towards the scientific and quantitative direction. In contrast, China's investment in this area is obviously inadequate. It is common that we analyze by coaches' eyes and predict by experience, which limits the further improvement of athletes' score.

Accurate analysis of athletes' video is an effective technical way to improve the training effect, and the realization of tracing of athletes in the technical video is the important basis of analyzing technical videos. So this article is aimed at applying the modern high-tech achievements in sports training and focus on how to achieve the tracing of athlete in sports technical video to help coaches to carry out a proper assessment of the technical characteristics of the athletes, in order to provide strong technical support for the improvement of athletes sports' performance.

\section{TECHNICAL PLAN}

To track athletes in sports technical video, we have to use not only a wealth of sports knowledge, but also the technology of crossing computer vision research. Nowadays the technique OpenCV(open source computer vision library supported by the Intel company) is good at image processing, pattern recognition, target detection and tracing, so this article tries to realize the tracing of athlete in sports technical video base on this technology ${ }^{[4-9]}$. What needs to be pointed out is that although there are many algorithms based on OpenCV technology platform, which can realize the athletes' tracing ${ }^{[10-12]}$, but any algorithm owns its own characteristics and weakness. So it is required to reasonably choose the appropriate algorithm to apply to athletes' state of motion in sports technical video.

The CamShift algorithm can effectively solve the target deformation and occlusion problems, and its demand on system resources is less, time complexity is low and could track the moving target well in simple background. So this article uses this algorithm to track the athletes in sports video. But what needs to be notified is that because that CamShift algorithm only takes care the color histogram and ignores the target's moving characteristic in space, when the background complexity or the disturbances and noise are the same, the tracing may fail. So we need to improve the CamShift algorithm appropriately, which means we need add the target prediction algorithm.

Kalman filter can overcome some of the goals blocking; mutual interference between the target, target high moving speed, some small similar background interference leading to tracing failure, so it is effective to predict the position of target and combined with CamShift algorithm, it is better for the tracing of moving target in the video. Combination of Kalman and CamShift algorithm mainly consists of three parts:

1) Kalman predicts the target's position in the current frame. Since the Kalman filter cound predict the next estimated value based on the previous observed value and estimated value, using Kalman filter could reliably predicts target's position in the current frame.

2) CamShift matching the moving target. Based on the color probability distribution of the moving target, in the neighborhood of Kalman filter estimated target position, using CamShift algorithm to search the similar target in the target template.

3) Update Kalman filter state. Using the current moving target's size and centroid as the Kalman filter ovservation 
value to update the Kalman filter's state, then to estimate the target's estimated value in the next frame more precisely.

\section{RESUlt AND DiscUSSION}

Figure 1 and Figure 2 are tracking effect figures using CamShift algorithm and improved CamShift algorithm separately. Through comparing the three graphs in Figure 1 we can find that: Camshift algorithm can track targets well in stable background and when the environment light is changing slowly and speed of moving target is not so high. In complex situation through adjusting the threshold value, the correctness of tracking can also be guaranteed, but because the color of background and moving target is quite similar, the tracking window becomes also larger and larger.

This is mainly because CamShift algorithm loses the moving target in tracking and regards this target, as a new moving target in the subsequent calculations, which results the number of detected targets, is larger than the actual one. In addition, the detection area is quite larger than the actual moving target, which causes the detection area become larger and larger and finally to be the whole figure. However, when we use Kalman filter prediction, the searching area is reduced, the error rate also dropped significantly and the size of detecting box is also appropriate.

In conclusion, when combing the Kalman prediction and CamShift tracking algorithm, athletes in technical videos can be effectively tracked and this technology plan can be used as reference for other related researches.

\section{CONCLUSION}

After accurate analysis of athletes" technical videos, we can give correct assessment of athletes' technical characteristics and provide important basis for making scientific training mission for athletes.However, realizing the tracing of athletes in technical videos is the important foundation of analyzing technology precisely.

This article combines the Kalman prediction and CamShift tracing algorithm and realizes the athletes' tracing in technical videos based on OpenCV technology. The case study shows that when compared CamShift algorithm, the improved CamShift tracing algorithm has less searching area, significantly reduced error rate, appropriate detection box size, and it can effectively track athletes in the technical video. The plan proposed in this article can provide case reference for technology study in sports training.

\section{ACKNOWLEDGE}

This work was supported by the program of Soft Science of Science\&Technology department of SiChuan Province. [grant number:2012ZR0096 and TY2012206] And, the research received the grant from the funding of Laboratory and Equipment Management of SWJTU[grant number:201234]. And supported by the Fundamental Research Funds for the Central Universities,[grant number:SWJTU11BR121and122]

\section{REFERENCES}

[1] Xu DaCheng, Zhang MingHui, Bao ChengGong, The Queries for Utilization of Run-up Speed of Chinese Long Jump Athletes, Journal of PLA institute of physical education, 2010 29(4):26-30

[2] Yu ShuXiang, Shen JunQi, Kinematics Analysis of Take-off Buffer and Pedal Stretch Stage of Liaoning Outstanding Young Woman Long Jump Athletes, Journal of ShenYang sport university, 2011 30(5):108-110

[3] Li ZhaoWen, Research review of Long jump technology, Sports Research and Education, 2011 26:184-185

[4] William K. Pratt, Digital Image Processing, BeiJing: China Mechine Press, 2005

[5] Zhao ChunHui, Pan Quan, Liang Yan, Video Imagery Moving Targets Analysis, BeiJing: National Defence Industry Press, 2011

[6] Shen TingZhi, Yan XueMei, Digital Image Processing and Pattern Recognition, BeiJing: Beijing Institute of Technology Press, 2007

[7] Guo WenQiang, Hou YanYOng, Digital Image Processing, Xi'An: XiDian University Press, 2009

[8] Tian Yan, Peng FuYuan, Digital Image Processing and Analysia, WuHan: Huazhong University of Science \& Technology Press, 2009

[9] Dong ShiChong, Wang TianZhen, Xu Gang, Motion Detection in Video Images, Journal of WuHan university of technology, 2004 26(4):1-3

[10] Liu Rui Zhen, Yu ShiQi, OpenCV Tutorial, Beijing: BeiHang University Press, 2007

[11] Hu Lei, Application and analysis about some arithmetics for image denoising, Information Technology, 2007 31(7):81-83

[12] Peng YanFang, The research on detection and traeking algorithms of VideoMotio .Objects, WuHan: WuHan University of Technology, 2010

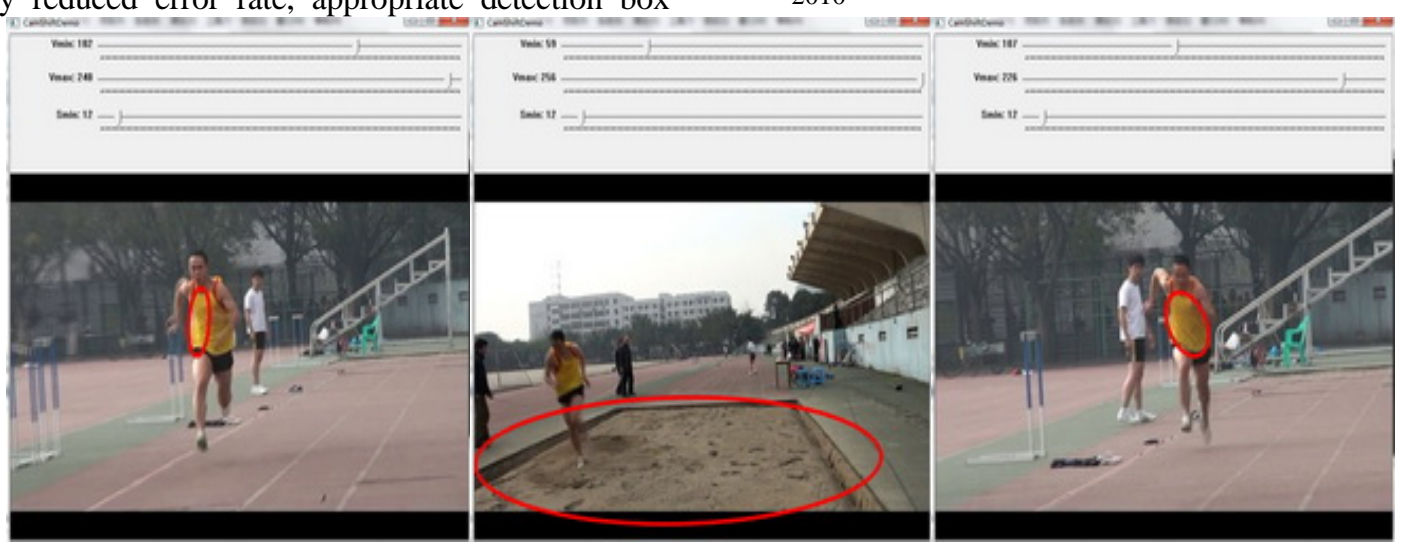

Figure 1. CamShift algorithm training effect 


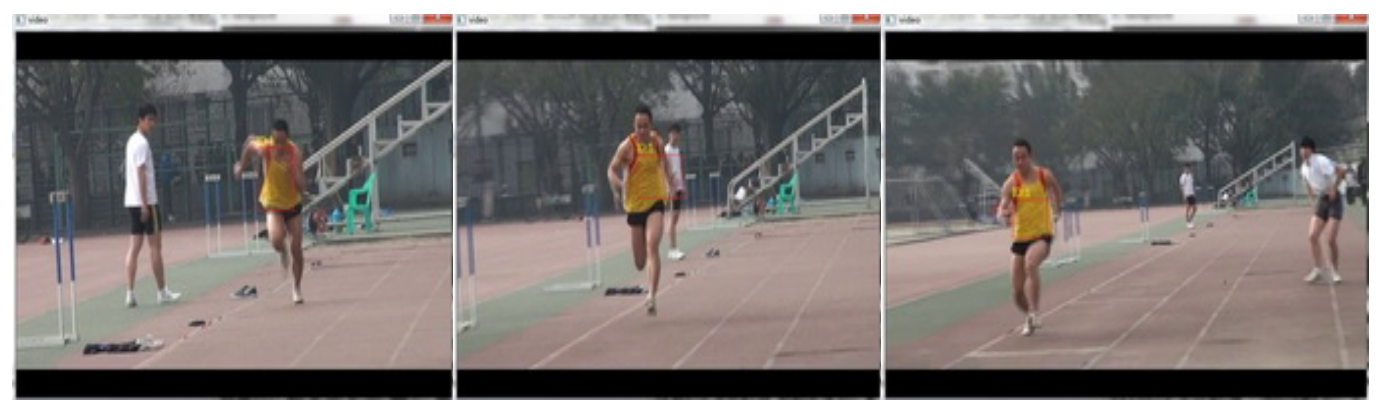

Figure 2. CamShift algorithm training effect 